\title{
Intervenção Psicológica no Acompanhamento Hospitalar de uma Criança Queimada
}

\author{
Psychological Intervention in the Hospital \\ Accompaniment of a Burnt Child \\ Intervención Psicológica en el Acompañamiento \\ Hospitalario de un Niño Quemado
}

Adriano Valério dos

Santos Azevêdo \& Ana

Flávia Trindade dos

Santos

Universidade

Federal de Sergipe
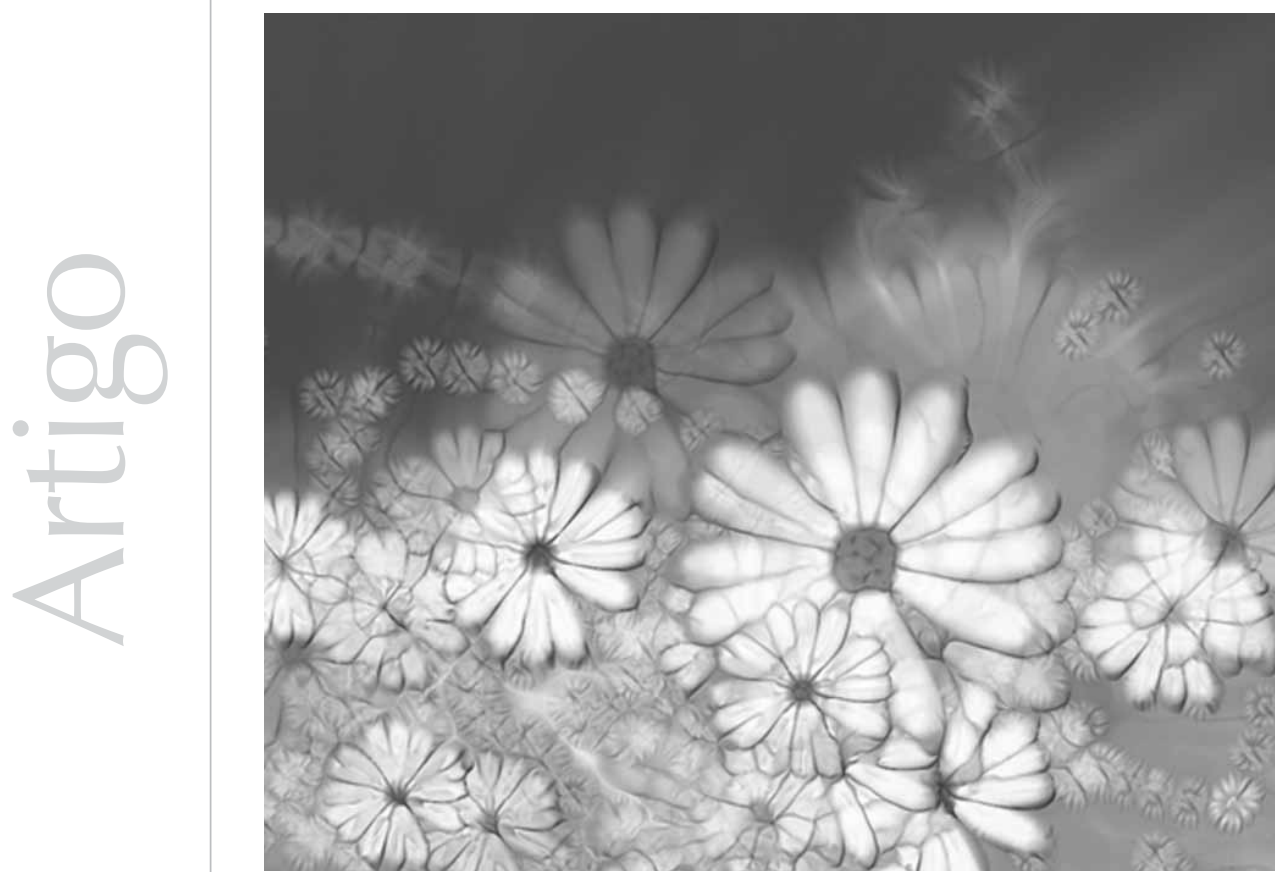
Resumo: A teoria cognitivo-comportamental destaca a interação de processos cognitivos, afetivos e comportamentais no funcionamento psicológico. O presente estudo objetivou analisar um modelo de intervenção psicológica no acompanhamento hospitalar de uma criança queimada. Foram utilizadas as seguintes técnicas: entrevista motivacional, mapa de rosto de sentimentos, jardim de flor de pensamentos, Escala Numérica de Relato Verbal para Avaliação da Dor, treino de relaxamento e informações psicoeducativas. A análise de dados indicou que a intervenção se mostrou efetiva e que a criança desenvolveu habilidades adaptativas que contribuíram para a adesão ao tratamento na unidade hospitalar e para o manejo da dor. Recomenda-se a aplicação dessas técnicas de intervenção nas unidades de pediatria hospitalar para verificar a necessidade de adaptações. Acredita-se que as intervenções cognitivo-comportamentais podem ser utilizadas no ambiente hospitalar devido às suas contribuições para a recuperação da saúde integral da criança hospitalizada.

Palavras-chave: Intervenção psicológica. Criança hospitalizada. Queimaduras. Saúde infantil.

Abstract: The behavioral-cognitive theory highlights the interaction of cognitive, affective and behavioral processes in the psychological functioning. The present study had as objective the analysis of a model of psychological intervention in the hospital accompaniment of a burnt child. The following techniques were used: motivational interview, face map of feelings, flower garden of thoughts, Numerical Scale for Pain Evaluation, relaxation training, and educative information. The analysis of the data indicated that the intervention was effective, that the child developed adaptative abilities that contributed to the adhesion to the treatment in a hospital unit and to the handling of pain. It is recommended the use of these techniques of intervention in the units of hospital pediatrics to verify the necessity of adaptations. We believe that the behavioral-cognitive intervention can be used in the hospital environment, due to its contributions for the recovery of the hospitalized child integral health.

Keywords: Psychological intervention. Hospitalized child. Burns. Child health

Resumen: La teoría cognitivo comportamental destaca la interacción de procesos cognitivos, afectivos y de comportamiento en el funcionamiento psicológico. El presente estudio objetivó analizar un modelo de intervención psicológica en el acompañamiento hospitalario de un niño quemado. Fueron utilizadas las siguientes técnicas: entrevista motivacional, mapa de rostro de sentimientos, jardín de flor de pensamientos, Escala Numérica de Relato Verbal para Evaluación del Dolor, entrenamiento de relajación, y las informaciones psico-educativas. El análisis de datos indicó que la intervención se mostró efectiva, el niño desarrolló habilidades adaptativas que contribuyeron para la adhesión al tratamiento en la unidad hospitalaria y manejo del dolor. Se recomienda la aplicación de estas técnicas de intervención en las unidades de pediatría hospitalaria para verificar la necesidad de adaptaciones. Se cree que las intervenciones cognitivas de comportamiento pueden ser utilizadas en el ambiente hospitalario, debido a sus contribuciones para la recuperación de la salud integral del niño hospitalizado.

Palabras clave: Intervención psicológica. Niño hospitalizado. Quemaduras. Bienestar del nino.

Os principais estudos na área da Psicologia pediátrica destacaram as reações das crianças provocadas pelo processo de hospitalização, tais como mudanças no humor, sensações de medo, regressões no comportamento, agressividade, transtornos do sono e déficit nas habilidades sociais, considerando relevante a presença do acompanhante, representada com frequência pela genitora, que compartilha com o filho momentos de ansiedade (Barros, 1999; Crepaldi, Rabuske, \& Gabarra, 2006; Lindquist, 1993; Quiles \& Carrilo, 2000; Roberts \& Steele, 2009; Seagull, 2000). Dessa forma, o período relacionado à hospitalização durante as primeiras fases do desenvolvimento humano repercute nos aspectos cognitivos, emocionais e comportamentais da criança.
A situação de adoecimento e hospitalização ocasiona na criança sofrimento emocional e físico decorrente das características do ambiente hospitalar, da sensação de abandono, da perda da identidade e da ruptura do núcleo familiar (Camon, 2006; Chiattone, 2003; Dias, Baptista, \& Baptista, 2003). Na hospitalização para o tratamento de queimaduras, a criança pode desenvolver sensações de medo em virtude dos procedimentos invasivos que são utilizados na área queimada pela equipe de saúde.

A queimadura representa uma lesão provocada por agentes externos que incluem o fogo, o choque elétrico, os produtos químicos e os demais fatores que danificam a pele e 
suas estruturas internas (Borges, 1995). A área lesionada deve ser avaliada conforme sua profundidade, a partir das classificações de $1 \stackrel{0}{ }, 2^{\circ}$ e $3^{\circ}$ graus, sendo as duas últimas consideradas mais graves, e quanto à extensão, atribuindo-se um escore quantitativo que se relaciona entre pequeno, médio e grande queimado.

Destaca-se que a dor representa um fenômeno vivenciado pelos pacientes hospitalizados com queimaduras. A International Association for Study of Pain (IASP, 1979) ressaltou que as sensações dolorosas estão ligadas a uma lesão nas áreas do corpo que é percebida de maneira distinta pelas pessoas. No que se refere ao fenômeno da dor, a percepção do indivíduo acerca de uma sensação desagradável no corpo envolve a interação de fatores fisiológicos e psicológicos. A noção de doença parte do pressuposto de que a mente e o corpo estão integrados na vivência do indivíduo e desempenham um papel relevante na manutenção e na evolução do quadro clínico, de tal modo que as repercussões psicológicas de uma experiência ocasionam o surgimento de uma doença física (Mello-Filho, 2002) ou as vivências em situações traumáticas provocam reações corporais e psicológicas no indivíduo.

Na literatura consultada, os autores destacaram a importância de reconhecer o papel das emoções, considerando que as sensações desagradáveis no corpo integram um conteúdo psicológico quando a criança começa a expressar os afetos por meio dos impulsos agressivos como o choro e o grito de dor física e de sofrimento emocional (Ballone, 2007; Jeammet, Reynaud, \& Michel, 2000). Outras pesquisas destacaram a importância de compreender os sentimentos do paciente queimado diante das sensações de dor e ansiedade, devido aos estressores físicos provenientes das mudanças fisiológicas do corpo, e dos estressores psicológicos que repercutem nas alterações do humor e comportamento (Carlucci, Rossi, Ficher,
Ferreira, \& Carvalho, 2007; Weinberg, Birdsall, Vail, Marano, Petrone, \& Mansour, 2000). Os pacientes hospitalizados com queimaduras podem apresentar dificuldades no manejo da dor, tendo em vista que as sensações dolorosas podem ser percebidas com intensidade mesmo diante de lesões no corpo que já se encontram cicatrizadas. Assim, considera-se fundamental o atendimento psicológico para pacientes que apresentam sofrimento psíquico secundário na presença de uma doença física.

A teoria cognitivo-comportamental focaliza a identificação, a análise e a modificação dos processos mentais relacionados ao pensamento, aos sentimentos e ao comportamento do indivíduo por meio de um enfoque educativo, com diferentes técnicas terapêuticas para promover mudanças cognitivas e comportamentais (Beck, 1976). O princípio central baseia-se no funcionamento dinâmico da cognição proveniente das interpretações que os indivíduos atribuem as suas experiências. As aplicações das intervenções cognitivo-comportamentais demonstraram eficácia para o tratamento de diversos transtornos clínicos, e a sua utilização nos ambientes de saúde, em pacientes com doenças crônicas, tais como câncer (Maguire, Hopwood, Tarrier, \& Howell, 1985; Scott, 1994; Worden, 1987), síndrome do intestino irritável (Schwartz \& Blanchard, 1986) e artrite reumatóide (Bradley, 1985) apontou os avanços que ocorreram na intervenção em Psicologia da saúde. Dados positivos indicaram que os pacientes apresentaram qualidade de vida, adaptação e enfrentamento das condições adversas das doenças, considerando a importância de novas pesquisas para verificar quais estratégias de intervenção podem ser desenvolvidas. O estudo teórico de Ritvo, Irvine, Katz e Shaw (2003) apresentou a estrutura básica de um programa de grupo para o atendimento de pacientes em tratamento médico, sendo o principal foco a promoção da saúde e o desenvolvimento de habilidades adaptativas. 
Poucas publicações se referiram ao uso dessas intervenções em pacientes hospitalizados, e alguns estudos enfatizam as sessões de grupo (Browder, 2003; Freeman, Schrodt, Gilson, \& Ludgate, 1993) e individuais (Blackburn, 1994; Scott, 1994; Walker \& Healy, 1980). Os dados destacaram as contribuições do atendimento psicológico hospitalar, mas o número limitado de amostras provenientes de autores internacionais revela a necessidade de mais pesquisas na área. A importância dos planos de tratamento, a formulação de casos clínicos, a aplicação de técnicas de psicoeducação e relaxamento e os registros das sessões, acompanhando os resultados obtidos, representam os principais pontos destacados nesses estudos. É importante ressaltar que, na literatura (Gorayeb \& Guerrelhas, 2003; Myiasaki, Domingos, \& Caballo, 2001), as recomendações para a atuação do psicólogo nos ambientes hospitalares priorizam o desenvolvimento de pesquisas e a avaliação constante das intervenções. Verifica-se a relevância de analisar as técnicas utilizadas pelo psicólogo no hospital para estabelecer diretrizes que possam facilitar a atuação profissional visando a contribuir para a sistematização das intervenções.

Este estudo objetivou analisar um modelo de intervenção psicológica utilizado no acompanhamento hospitalar de uma criança queimada. O termo modelo integra concepções teóricas e um conjunto de técnicas e procedimentos para orientar o planejamento e a avaliação das intervenções. Acredita-se que o indivíduo hospitalizado precisa desenvolver estratégias para auxiliar a adesão ao tratamento e a reinserção no ambiente familiar e social. Foram incluídas, nessa proposta de intervenção, técnicas cognitivo-comportamentais para crianças. Contribuições teóricas de Bandura (1962) acerca da aprendizagem social pela observação, os estudos sobre técnicas de mudanças comportamentais elaboradas por Skinner (1995) e os avanços na teoria cognitiva (Beck \& Alford, 2000) acerca do intermédio da cognição nos processos psicológicos permitiram delimitar as estratégias de intervenção.

O presente artigo descreve um relato de pesquisa proveniente da atuação no Serviço de Psicologia do Hospital de Urgências de Sergipe (HUSE), na Unidade de Tratamento de Queimados (UTQ). Utilizou-se o delineamento de estudo de caso com base nos procedimentos utilizados no processo de intervenção psicológica.

\section{Método}

\section{Participante}

Procurou-se ilustrar o caso clínico por meio de um nome fictício, Laurence, sexo feminino, 07 anos, hospitalizada na UTQ, vítima de queimaduras de $2^{\circ}$ e $3^{\circ}$ graus nos membros inferiores, com o diagnóstico de grande queimada. A equipe de saúde dessa unidade hospitalar solicitou um parecer psicológico que descrevesse os seguintes comportamentos da criança: manifestações de choro, queixas frequentes relacionadas a dor nas áreas do corpo que estavam em processo de cicatrização e dificuldade de adaptação nos procedimentos técnicos do setor. Iniciouse a discussão desse caso clínico com a equipe de saúde para, em seguida, realizar o planejamento das intervenções.

\section{Instrumentos}

No acompanhamento psicológico hospitalar, foram utilizadas as seguintes técnicas: entrevista motivacional (Stallard, 2007), mapa de rosto de sentimentos (Bernard \& Joyce, 1984), jardim de flor de pensamentos (Friedberg \& Mcclauren, 2004), Escala Numérica de Relato Verbal para Avaliação da Dor (Greenberger \& Padesky, 1999), treino de relaxamento autógeno (Shultz, 1967) com a respiração diafragmática e as informações psicoeducativas relativas às orientações para a criança acerca de procedimentos da unidade hospitalar, visando a facilitar a adesão ao tratamento. 


\section{Procedimentos}

Foram realizados 24 atendimentos psicológicos com a criança em uma enfermaria de queimados com periodicidade semanal em dias alternados, incluindo a participação da acompanhante, representada pela genitora, e a equipe de saúde. Destaca-se que o acompanhamento psicológico hospitalar apresenta peculiaridades tais como: a ocorrência do atendimento em qualquer espaço do hospital; o número de sessões dependente dos objetivos definidos pelo psicólogo; os dias, os horários e o tempo destinados ao atendimento podem sofrer variações; o contrato terapêutico é realizado nas entrevistas iniciais, mas a continuidade do atendimento depende exclusivamente do período em que o paciente permanece hospitalizado, e o atendimento psicológico pode ter início a partir da solicitação da equipe de saúde ou por meio das atividades de rotina do psicólogo hospitalar, quando este identifica essa demanda diante da sua integração permanente em uma equipe multiprofissional.

A criança permaneceu hospitalizada durante três meses, e o processo de intervenção ocorreu a partir do segundo mês de hospitalização, sendo esse vínculo finalizado no momento da alta hospitalar, totalizando oito semanas de atendimento psicológico. Buscou-se estabelecer o contato inicial com a equipe de saúde e com a genitora para obter informações sobre a criança. A entrevista motivacional desenvolvida por Stallard (2007), que tem o objetivo de identificar processos de mudança no indivíduo, foi utilizada para conhecer a percepção da criança acerca da situação de adoecimento, e foi realizada por meio do relato verbal e da solicitação de um desenho, buscando identificar seu interesse para promover mudanças visando à adesão ao tratamento.

O mapa de rosto de sentimentos e o jardim de flor de pensamentos (Bernard \& Joyce,
1984; Friedberg \& Mcclauren, 2004) foram os instrumentos utilizados para estabelecer o vínculo com a criança e identificar as situações, os pensamentos, sentimentos e comportamentos de forma criativa. No mapa de rostos, solicitou-se à criança que desenhasse um círculo em uma folha em branco e fizesse uma expressão facial; em seguida, identificavase o sentimento atribuindo-lhe um escore de 0 a 10. Buscou-se também identificar o sentimento por meio do relato verbal da criança, e, em seguida, quantificá-lo no círculo em branco com lápis colorido.

No jardim de flor de pensamentos, a criança foi solicitada a desenhar um solo que representava uma situação em que o talo indicava o pensamento, e a flor, o sentimento, registrandose em seguida o seu comportamento, e, com o lápis colorido, era ilustrado o desenho, o que permitiu facilitar seu entendimento acerca da técnica. Outro instrumento utilizado foi uma escala numérica de 0 a 10, adaptada do modelo de Greenberger e Padesky (1999) para avaliar os níveis da dor (leve, médio, intenso) através do relato verbal da criança, quando esta identificava a área do corpo avaliando o nível da dor com a escala.

Utilizou-se o treino de relaxamento autógeno (Shultz, 1967) para tensionar e relaxar partes do corpo da criança por meio da sua imaginação; inicialmente, a criança foi solicitada a identificar uma região do corpo e a imaginar sensações de dor e, em seguida, de ausência de dor. Optou-se por essa estratégia devido à situação atual que se encontrava a criança, que sentia dificuldade de lidar com a dor. Com maior frequência, foi utilizada a respiração diafragmática, que consiste em focalizar o processo de respiração para minimizar tensões nas esferas fisiológicas e psicológicas, quando o indivíduo começa a identificar as sensações no corpo. A respiração se torna mais lenta e a pressão sanguínea diminui, propiciando efeito calmante, principalmente quando são apresentadas frases que levam o 
De

acordo com

Guimarães

(2001), 0

exercício de

relaxamento

possibilita a

identificação,

pelo paciente,

dos níveis de

inspiração e

de expiração,

assim como da

percepção das

sensações do

organismo. paciente a perceber o relaxamento do corpo, procedimento realizado nesse estudo de caso.

De acordo com Guimarães (2001), o exercício de relaxamento possibilita a identificação, pelo paciente, dos níveis de inspiração e de expiração, assim como da percepção das sensações do organismo. Foram apresentadas as seguintes instruções para a criança: feche os olhos e preste atenção no ar que entra e sai do seu corpo; imagine um balão no seu abdômen, e, quando você inspirar, estará enchendo-o, e, ao expirar lentamente, pense que você está apagando uma vela e sentindo o seu corpo relaxado. A criança foi orientada a praticar a respiração diafragmática, principalmente nas situações que envolviam os procedimentos de rotina do setor, tais como a realização de curativos na enfermaria hospitalar. A genitora também foi orientada para acompanhar a criança durante esse exercício de respiração.

Utilizaram-se as informações psicoeducativas, informando à criança a importância de continuar com o tratamento da queimadura na unidade hospitalar, com o objetivo de facilitar sua integração nos procedimentos do setor e de fortalecer os vínculos com o acompanhante e com equipe de saúde. As orientações à genitora foram dadas visando a facilitar o manejo das situações vivenciadas no período de hospitalização com a criança, priorizandose a expressão dos vínculos afetivos, o apoio e o reforço de comportamentos adaptativos da criança, com base nas estratégias para orientação de pais utilizadas na abordagem cognitivo-comportamental.

A publicação deste estudo foi aprovada pelo Comitê de Ética e Pesquisa com Seres Humanos da instituição hospitalar. A estrutura metodológica do estudo de caso foi fundamentada em uma investigação empírica, por meio de um conjunto de técnicas e de procedimentos que visavam à geração de dados e à busca de evidências, pontos recomendados por Yin (2004). Trata-se de um caso relevante para estudo por ser único e ainda não evidenciado na literatura científica, o que justifica o delineamento utilizado para investigação.

\section{Resultados}

Diante das informações obtidas com a equipe de saúde, buscou-se estabelecer o contato com a genitora, que se mostrou receptiva ao diálogo destacando as reações de choro e tristeza da criança, o que indicava que percebia a importância do acompanhamento psicológico. A criança apresentava um déficit de habilidades na expressão verbal, mas relatou que vivenciava períodos intensos de dor provocados pelos procedimentos realizados na unidade hospitalar, sendo que as sensações dolorosas se iniciavam após a realização do curativo no centro cirúrgico.

$\mathrm{Na}$ entrevista motivacional, foram abordadas questões acerca da percepção da criança no que se refere à situação de adoecimento e hospitalização, assim como seu interesse para iniciar mudanças visando à adesão ao tratamento. A criança desenhou sua imagem corporal em uma folha em branco, destacando os membros inferiores comprometidos pela queimadura, que impossibilitavam sua locomoção, e mostrou-se cooperativa ao relatar seu interesse para participar do tratamento das queimaduras. As situações de crise geralmente ocorriam durante o período da manhã, com o choro prolongado que representava a intensidade da dor. Assim, buscou-se permanecer ao lado da criança para entender essa vivência por meio de uma compreensão empática, e esse procedimento possibilitou desenvolver o processo de vinculação entre o psicólogo e a díade (mãe/criança).

O diagnóstico da queimadura indicava um tratamento longo devido às lesões na pele que necessitavam de cirurgia plástica de enxerto para recomposição das áreas lesionadas. Destaca-se que algumas áreas do corpo 
estavam cicatrizadas, mas a criança indicava a presença de sensações dolorosas no membro inferior esquerdo. Dessa forma, foi possível perceber que a criança apresentava repercussões fisiológicas e psicológicas da dor, provavelmente oriundas da experiência traumática provocada pela queimadura. Essas pressuposições foram compartilhadas com os profissionais de saúde da unidade hospitalar, que demonstraram interesse nessa informação.

No contato interdisciplinar com a fisioterapeuta, iniciou-se um breve relato do caso clínico, diante do fato de as queixas principais estarem relacionadas com a dificuldade da criança no procedimento fisioterapêutico e diante da necessidade que a profissional manifestou para o aprimoramento da sua relação no atendimento infantil. Com os demais integrantes da equipe de saúde, procurou-se compartilhar informações educativas sobre a hospitalização infantil, destacando a importância do contato empático desses profissionais na relação com a criança, para que fosse possível compreender as reações psicológicas provenientes do processo de hospitalização. As técnicas de intervenção foram utilizadas com aplicações criativas para facilitar o entendimento da criança acerca das relações entre pensamentos, sentimentos e comportamentos. A transcrição dos dados, a verificação e a comparação dos escores representaram os principais critérios para a correção dos instrumentos.

A Tabela 1 apresenta uma síntese dos dados obtidos no acompanhamento psicológico. Verificou-se que, na primeira semana, a criança demonstrou receptividade durante a aplicação das técnicas. Utilizou-se o mapa de rosto de sentimentos no início de cada atendimento psicológico para identificar as emoções e, em seguida, o jardim de for de pensamentos para destacar as situações típicas vivenciadas pela criança, como, por exemplo, a realização de curativos e a sua forma de comunicação com a equipe de saúde. O treino de relaxamento autógeno foi utilizado nas fases iniciais dos atendimentos, assim como a respiração diafragmática, que foi mantida durante o período de acompanhamento hospitalar. A criança relatou as sensações agradáveis, e destaca-se que não foi evidenciada a presença de dor no corpo; assim, essas técnicas promoveram a distração, a capacidade de lidar com a dor e o controle da respiração.

Tabela 1. Dados obtidos no acompanhamento psicológico

\section{1ํㅗㄴ semana}

A criança demonstrou interesse para participar do tratamento das queimaduras/ Identificou pensamentos, sentimentos e comportamentos/Mostrou-se receptiva à técnica de relaxamento/ Identificou os níveis da dor com a escala numérica

\section{$2^{\underline{a}}-3^{\underline{a}}$ semanas}

A criança apresentou receptividade com a equipe de saúde/ Capacidade de lidar com a dor/ Adesão aos procedimentos do setor relacionados aos curativos e à fisioterapia/ Reestruturou pensamentos e compreendeu as informações psicoeducativas

\section{$4^{\mathrm{a}}-8^{\underline{a}}$ semanas}

A criança desenvolveu habilidades adaptativas durante a fisioterapia e na fase pós-cirúrgica/ Entendeu a existência de áreas cicatrizadas no corpo/ Apresentou mudanças positivas no humor e no comportamento

Entre a segunda e a terceira semanas, a criança estabeleceu comunicação com a equipe de saúde, e isso contribuiu para a adesão ao tratamento e para a reestruturação de pensamentos relacionados aos procedimentos técnicos do setor. Verificou-se que as intervenções da fisioterapia e os curativos 
diários despertavam na criança sensações de medo que ocasionavam a dor no corpo. Dessa forma, procurou-se registrar esses eventos com o uso da técnica do jardim de flor de pensamentos, o que possibilitou iniciar um questionamento com a criança visando a atribuir respostas adaptativas, auxiliando sua preparação para o enfrentamento dessas situações. Em uma sessão, Laurence identificou a seguinte situação: "O exercício da fisioterapeuta", o pensamento: "Eu não quero fazer, vai doer"; sentimento: "medo"; comportamento: "Fica chorando através de gritos". A criança foi solicitada a indicar o local doloroso atribuindo o escore (0-10) por meio da Escala Numérica de Relato Verbal, e foram discutidas as contribuições desse procedimento para sua recuperação. Laurence descreveu a seguinte resposta adaptativa: "Pode doer um pouco, mas vai passar, eu quero ir para minha casa e escola". Nesse momento, a criança identificou a situação geradora de medo, e, a partir disso, foi possível promover a reestruturação cognitiva com mudanças na maneira de perceber os eventos, tendo ela considerado importante a realização dos procedimentos técnicos da unidade hospitalar.

O desenvolvimento de habilidades adaptativas durante a fisioterapia predominou entre a quarta e a oitava semanas, assim como no período pós-cirúrgico, quando precisou ficar durante três dias em uma posição específica para contribuir com os resultados da cirurgia plástica de enxerto, realizada na região do glúteo para recomposição das áreas lesionadas. Em outros momentos, a criança identificava o nível da dor durante a fisioterapia e enfrentava o procedimento atribuindo respostas adaptativas que foram desenvolvidas no acompanhamento psicológico. Um ponto importante é o da inexistência de queixas sobre a dor no membro inferior do corpo, devido a sua percepção de que o local estava cicatrizado. Existem evidências que comprovam sua integração no tratamento e o entendimento da evolução clínica, o que representou um ponto positivo na sua recuperação. Os dados acerca da aplicação de técnicas e da evolução da criança estão destacados na Tabela 2.

Tabela 2. Síntese comparativa do Acompanhamento Psicológico (AP)

\section{Técnicas}

Mapa de rostos

Escala Numérica de Dor

\section{Início}

Sentimentos de tristeza (6)

Dor intensa (8)
No decorrer do AP

Sentimentos de alegria (6-8)

Dor leve (3)

\section{Características da criança}

Fase inicial

Choro constante

Dificuldades de adaptação

Vivência de sensações dolorosas

Queixas de dor em áreas específicas do corpo
No decorrer do AP

Receptividade com a equipe de saúde

Adesão ao tratamento

Capacidade de lidar com a dor

Entendimento acerca das áreas cicatrizadas

Deve-se ressaltar que, no decorrer dos atendimentos psicológicos, ocorreram mudanças positivas no humor da criança, e, na escala numérica, verificou-se redução dos níveis de dor. A criança desenvolveu estratégias cognitivas e comportamentais adaptativas que contribuíram para a vivência no período de hospitalização, se comparadas às queixas iniciais que indicavam sofrimento psicológico. O entendimento acerca das sensações dolorosas, representado pela atribuição de um novo significado a essa experiência, teve início a partir da percepção da criança quanto às reações 
fisiológicas e psicológicas da dor. A técnica de relaxamento permitiu desenvolver a capacidade de reflexão na criança acerca de reações corporais, e a avaliação dos níveis da dor, com o auxílio da genitora, possibilitou integrar a criança no automonitoramento, atividade que consistia no relato verbal da criança ao avaliar a dor e compartilhar com a genitora o escore da escala numérica.

Durante situações de crise, por meio do choro e gritos, a criança foi solicitada a identificar o local da dor indicando o escore, o que permitiu compreender e enfrentar a sensação dolorosa. Após a realização do curativo no centro cirúrgico, procurou-se ressaltar para a criança, por meio de um enfoque educativo, que as sensações dolorosas no corpo eram temporárias e oriundas da substância tópica que havia sido utilizada na área queimada, informações essas obtidas com a equipe de saúde.

O choro foi minimizado após as informações psicoeducativas dadas à criança. A psicoeducação foi utilizada para destacar a importância de a criança participar dos procedimentos do setor estabelecendo o contato com a equipe de saúde, o que permitiu facilitar a adesão para a continuidade do tratamento na unidade hospitalar. A genitora recebeu orientação adequada para lidar com as situações de crise da criança, que visava a demonstrar apoio e a compartilhar atividades lúdicas para facilitar a vivência da díade no brincar. Destaca-se o vínculo seguro no relacionamento da díade por meio da expressão dos afetos e do diálogo, um ponto importante que contribuiu para a evolução do acompanhamento psicológico.

\section{Discussão}

O principal interesse deste estudo foi analisar um modelo de intervenção psicológica utilizado no acompanhamento hospitalar de uma criança queimada. Neste estudo, o planejamento das intervenções terapêuticas foi realizado a partir da identificação dos problemas vivenciados pela criança hospitalizada, por meio do contato com a equipe de saúde e genitora. A escala numérica facilitou identificar a área do corpo e o nível da dor através do escore, e esse procedimento foi utilizado pela criança sem que apresentasse dificuldades, o que possibilitou iniciar o processo de atribuição de novos significados para as sensações dolorosas. Saber lidar com a dor representa um aspecto relevante que deve ser utilizado pelo paciente queimado (Mocelin \& Andreoli, 2008), com o auxílio de técnicas específicas e o apoio da equipe de saúde. Nesse contexto, o treino de relaxamento contribuiu de maneira significativa para inserir a criança na identificação e no controle da respiração.

As intervenções permitiram auxiliar a criança a identificar pensamentos, sentimentos e comportamentos, e as técnicas do mapa de rosto de sentimentos e jardim de flor de pensamentos mostraram-se adequadas no que se refere a sua aplicabilidade no ambiente hospitalar devido aos conteúdos lúdicos. As informações psicoeducativas sobre a importância da continuidade do tratamento das queimaduras permitiram facilitar o preparo da criança para enfrentar os procedimentos técnicos do setor. As pesquisas indicaram que a preparação psicológica para o enfrentamento das situações no hospital contribui para o desenvolvimento das habilidades adaptativas da criança e do acompanhante (Doca \& Costa-Júnior, 2007; Ribeiro, Tavano, \& Neme, 2002; Salmon \& Pereira, 2002).

Os estudos sobre as aplicações das intervenções cognitivo-comportamentais destacaram a importância de utilizar um enfoque educativo para facilitar a compreensão do indivíduo acerca da situação de adoecimento (Blackburn, 1994; Browder, 2003; Scott, 1994). Em um estudo de caso clínico com uma criança hospitalizada na unidade de queimados, Walker e Healy (1980) utilizaram 
estratégias cognitivo-comportamentais e ressaltaram que as técnicas de modificação de comportamento, treino de relaxamento, biblioterapia e atividades lúdicas apresentaram resultados positivos. Stoddard, Sheridan, Saxe, King, Chedekel, Schnitzer, e Martyn (2002) destacaram que os estudos acerca dos efeitos das intervenções cognitivo-comportamentais em crianças hospitalizadas estão em constante processo de avaliação, principalmente devido às contribuições das técnicas no que se refere a lidar com a dor e com as situações de estresse. Dessa forma, existem evidências que comprovam a efetividade dessas intervenções com crianças hospitalizadas, e os dados do presente estudo confirmam a literatura consultada.

A utilização das intervenções psicológicas no acompanhamento hospitalar representou um recurso importante para a sistematização das técnicas e dos procedimentos. O estabelecimento do diálogo constante do psicólogo com os profissionais do setor possibilitou conhecer as características do ambiente hospitalar e as técnicas utilizadas pelos profissionais para o tratamento das queimaduras. Os estudos na área da Psicologia pediátrica indicaram que a comunicação entre o psicólogo e a equipe de saúde representa uma característica relevante para o planejamento e a avaliação do processo de intervenção com a criança (Crepaldi et al., 2006; Pedromônico, 2006). Blackburn (1994) destacou que as aplicações das intervenções cognitivocomportamentais no ambiente hospitalar apresentam similaridades com a metodologia de terapia cognitiva padrão. As principais diferenças incluem a maior frequência das sessões e a importância de compartilhar informações com a equipe de saúde, o que permite verificar os comportamentos emitidos pela criança e a evolução dos dados obtidos na intervenção. Esse procedimento representa um recurso bastante utilizado nessa abordagem teórica, o que foi destacado por Beck e Alford (2000) sobre a importância de considerar o contexto ambiental e as queixas destacadas pelos pacientes, que geralmente envolvem o relacionamento com outros indivíduos, por considerar que a inclusão de pessoas significativas amplia as perspectivas de intervenção psicológica.

Neste estudo, a análise de dados indicou que as intervenções se mostraram efetivas devido aos seguintes fatores: adesão da criança ao tratamento, pela sua participação nos procedimentos técnicos do setor, controle da dor, com a utilização da escala numérica, ausência de queixas dolorosas na área cicatrizada, receptividade junto à equipe de saúde e redução das situações de crise. As evidências deste estudo indicam que as intervenções cognitivo-comportamentais podem ser utilizadas com crianças hospitalizadas, desde que o psicólogo realize o planejamento dos instrumentos que serão aplicados baseando-se no problema inicial vivenciado pela criança. Para a aplicação das técnicas, é preciso utilizar a criatividade no contexto hospitalar, considerando que, nas enfermarias, existe a presença constante de pacientes e de profissionais de saúde. Recomenda-se a aplicação das técnicas em novas amostras para analisar as possibilidades de adaptações com o propósito de ampliar as pesquisas acerca dessa temática. Nesse contexto, Yin (2004) destacou que os estudos de caso permitem a construção de teorias, e a replicação do método possibilita avaliar os dados obtidos fazendo comparações com outras pesquisas, o que representa um procedimento adequado.

Em síntese, o objetivo do presente estudo foi alcançado, e, para outras pesquisas, seguem as seguintes sugestões: é necessário investigar se outras técnicas podem ser utilizadas e quais resultados podem ser obtidos com novas amostras, por se tratar de apenas um estudo de caso. A proposta apresentada neste relato de pesquisa integrou referencial teórico da psicossomática e da abordagem cognitivo-comportamental para ampliar as noções de adoecimento e as perspectivas 
de intervenção psicológica. No que se refere às limitações deste estudo, na fase de avaliação inicial, a dificuldade da criança em se expressar verbalmente impossibilitou a aplicação de instrumentos psicométricos para verificação dos níveis de estresse e depressão; assim, não foi possível fazer comparações de dados objetivos nas fases pré e pósintervenção, com exceção apenas da Escala Numérica de Avaliação da Dor e do mapa de sentimentos, que foram aplicados com êxito. É importante ressaltar que o atendimento psicológico focalizou apenas o período de hospitalização da criança, não tendo sido possível estabelecer sessões para verificação dos efeitos (follow up) após a alta hospitalar. Os dados obtidos no processo de intervenção foram provenientes dos escores de duas técnicas, das mudanças de comportamento apresentadas pela criança, assim como dos relatos identificados durante a aplicação das técnicas que envolviam reestruturação cognitiva e das considerações apresentadas pela genitora e pela equipe de saúde.

Destaca-se que o acompanhamento psicológico hospitalar na área pediátrica se torna efetivo quando o psicólogo desenvolve a capacidade de adaptação para utilizar as técnicas cognitivocomportamentais nesse ambiente repleto de variáveis, que se distinguem de um contexto clínico estruturado. Os dados obtidos destacaram que a utilização da metodologia no estudo de caso facilita delimitar técnicas e procedimentos, e isso contribui para o desenvolvimento científico da intervenção psicológica com crianças hospitalizadas.

\section{Adriano Valério dos Santos Azevêdo}

Mestre em Psicologia Social - Universidade Federal de Sergipe (UFS), Sergipe, SE-Brasil. Especialização em

Psicologia Hospitalar. Pesquisador da área da Psicologia da Saúde e Hospitalar.

E-mail:adrianoazevedopsi@yahoo.com

\section{Ana Flávia Trindade dos Santos}

Especialização em Psicologia Conjugal e Familiar. Serviço de Psicologia do Hospital de Urgências de Sergipe, Sergipe, SE- Brasil.

E-mail:flatrindade_se@hotmail.com

Endereço para envio de correspondência:

Universidade Federal de Sergipe, Núcleo de Pós-Graduação em Psicologia Social Cidade Universitária Prof.

Aloísio de Campos Av. Marechal Rondon s/n, Jardim Roza Elze

São Cristóvão, Sergipe - Brasil CEP 49100-000

Recebido 3/11/2009, 1a Reformulação 27/1/2011, Aprovado 4/4/2011

Referências
Ballone, G. J. (2007). Da emoção à lesão (2a ed.). São Paulo: Manole.

Bandura, A. (1962). Social learning through imitation. Lincoln, NE: University of Nebraska Press.

Barros, L. (1999). Psicologia pediátrica: perspectiva desenvolvimentista. Lisboa: Climepsi.

Beck, A. T. (1976). Cognitive therapy and the emotional disorders. New York: International Universities Press.

Beck, A. T., \& Alford, B. A. (2000). O poder integrador da terapia cognitiva. Porto Alegre: Artmed.

Bernard, M.E., \& Joyce, M.R. (1984). Rational emotive therapy whith children and adolescents. New York: Wiley.

Blackburn, I. M. (1994). Depressão severa em pacientes hospitalizados. In J. Scott, J. M. G. Williams, \& A. T. Beck
(Orgs.), Terapia cognitiva na prática clínica: um manual prático (pp. 01-30). Porto Alegre: Artmed.

Bradley, L. A. (1985). Effects of cognitive behavioural therapy on pain behaviour of rheumatoid arthritis patients: Preliminary outcomes. Scandinavian Journal of Behaviour Therapy, 14, 51-64.

Browder, A. S. W. (2003). Pacientes em ambientes de hospitalização parcial. In J. R. White, \& A. S. Freeman (Orgs.), Terapia cognitivo-comportamental em grupo para populações e problemas específicos (pp. 417-444). São Paulo: Roca.

Bernard, M. E., \& Joyce, M. R. (1984). Rational emotive therapy whith children and adolescents. New York: Wiley.

Borges, E. (1995). Queimaduras em crianças e intervenção psicológica. Porto Alegre: Artmed.

Camon, V. A. (2006). Psicologia hospitalar: teoria e prática. São Paulo: Pioneira Thomson Learning. 
Carlutti, V. D. S., Rossi, L. A., Ficher, A. M. F. T., Ferreira, E., \& Carvalho, E. C. (2007). A experiência da queimadura na perspectiva do paciente. Revista da Escola de Enfermagem USP, 41(1), 21-28.

Chiattone, H. B. C. (2003). A criança e a hospitalização. In V. A A. Camon (Org.), A psicologia no hospital (pp. 23-100). São Paulo: Pioneira Thomson Learning.

Crepaldi, M. A., Rabuske, M. M., \& Gabarra, L. M. (2006). Modalidades de atuação do psicólogo em psicologia pediátrica. In M. A. Crepaldi, M. B. M. Linhares, \& G. B. Perosa (Orgs.), Temas em psicologia pediátrica (pp. 13-55). São Paulo: Casa do Psicólogo.

Dias, R. R., Baptista, M. N., \& Baptista, A. S. D. (2003). Enfermaria de pediatria: avaliação e intervenção psicológica. In M. N. Baptista, \& R. R. Dias (Orgs.), Psicologia hospitalar: teoria, aplicações e casos clínicos (pp. 53-74). Rio de Janeiro: Guanabara.

Doca, F. N. P., \& Costa-Junior, A. L. (2007). Preparação psicológica para admissão hospitalar de crianças: uma breve revisão. Paidéia, 17(37), 167-179.

Freeman, A., Schrodt, G. R., Gilson, M., \& Ludgate, J. W. (1993). Group cognitive therapy with inpatients. In J. H. Wright, M. E. Thase, A. T. Beck, \& J. W. Ludgate (Eds.), Cognitive therapy with inpatients (pp. 121-153). New York: Guilford Press.

Friedberg, R. D., \& Mcclure, J. M. (2004). A prática clínica de terapia cognitiva com crianças e adolescentes. Porto Alegre: Artmed.

Gorayeb, R., \& Guerrelhas, F. (2003). Sistematização da prática psicológica em ambientes médicos. Revista Brasileira de Terapia Comportamental e Cognitiva, 5(1), 11-19.

Greenberger, D., \& Padesky, C. (1999). A mente vencendo o humor. Porto Alegre: Artmed.

Guimarães, S. S. (2001). Técnicas cognitivas e comportamentais. In B. Rangé (Org.), Psicoterapias cognitivo-comportamentais: um diálogo com a psiquiatria (pp. 113-130). Porto Alegre: Artmed.

International Association for the Study of Pain. (1979). Subcommitte on taxonomy of pain terms. A list with definitions and notes on usage. Pain, 6, 249-252.

Jeammet, P., Reynaud, M., \& Consoli, S. (2000). Psicologia médica. São Paulo: Medsi.

Lindquist, I. (1993). A criança no hospital: terapia pelo brinquedo. São Paulo: Scritta.

Maguire, P., Hopwood, P., Tarrier, N., \& Howell, T. (1985) Treatment of depression in cancer patients. Acta Psychiatrica Scandinavica, 320, 81-84.

Mello-Filho, J. (2002). Concepção psicossomática: visão atual. São Paulo: Casa do Psicólogo.

Myiasaki, M. C., Domingos, N. M., \& Caballo, V. E. (2001) Psicologia da saúde: intervenções em hospitais públicos. In B. Rangé (Org.), Psicoterapias cognitivo-comportamentais: um diálogo com a psiquiatria (pp. 463-474). Porto Alegre: Artmed.

Mocelin, V. L., \& Andreoli, P. B. A. (2008). Abordagem psicológica do paciente vítima de queimaduras. In E. Knobel, P. B. A. Andreoli, \& M. R. Erlichman (Orgs.), Psicologia e humanização: assistência aos pacientes graves (pp. 55-63). São Paulo: Atheneu.
Pedromônico, M. R. M. (2006). A relevância da avaliação psicológica na clínica pediátrica. In M. A. Crepaldi, M. B. M. Linhares, \& G. B. Perosa (Orgs.), Temas em psicologia pediátrica (pp. 81-107). São Paulo: Casa do Psicólogo.

Quiles, J. M. O., \& Carrillo, F. X. M. (2000). Hospitalización infantil. Repercusiones psicológicas. Teoria y práctica. Madrid: Biblioteca Nueva.

Ribeiro, R. M., Tavano, L. A., \& Neme, C. N. B. (2002). Intervenções psicológicas nos períodos pré e pós-operatório com pacientes submetidos a cirurgia de enxerto ósseo. Estudos de Psicologia, Campinas, 19(3), 67-76.

Ritvo, P. G., Irvine, M. J., Katz, J., \& Shaw, B. F. (2003). Pacientes em tratamento médico. In J.R. White, \& A. S. Freeman (Orgs.), Terapia cognitivo-comportamental em grupo para populações e problemas específicos (pp. 301-320). São Paulo: Roca.

Roberts, M. C., \& Steele, R. G. (2009). Handbook of pediatric psychology (4th ed.). New York: Guilford Press.

Salmon, K., \& Pereira, J. K. (2002). Predicting children's response to an invasive medical investigation: The influence of effortul control and parent behavior. Journal of Pediatric Psychology, 27(3), 227-233

Schultz, J. H. (1967). O treinamento autógeno. São Paulo: Mestre Jou.

Schwartz, S. P., \& Blanchard, E. B. (1986). Behavioural treatment of irritable bowel syndrome: A 1 year follow-up. Biofeedback Self Regulation, 11, 189-198.

Scott, J. (1994). Pacientes com câncer. In J. Scott, J. M. G. Williams, \& A. T. Beck (Orgs.), Terapia cognitiva na prática clínica: um manual prático (pp.125-154). Porto Alegre: Artmed.

Seagull, E. A. (2000). Beyond mothers and children: Finding the family in pediatric psychology. Journal of Pediatric Psychology, 25(3), 161-169

Skinner, B. F. (1995). Questões recentes na análise comportamental. Campinas, SP: Papirus

Stallard, P. (2007). Guia do terapeuta para os bons pensamentos - bons sentimentos: utilizando a terapia cognitivo comportamental com crianças e adolescentes. Porto Alegre: Artmed.

Stoddard, F. J., Sheridan, R. L., Saxe, G. N., King, B. S., King, B. H., Chedekel, D. S., Schnitzer, J. J., \& Martyn, J. A. J. (2002). Treatment of pain in acutely burned children. Journal of Burn Care \& Rehabilitation, 23(2), 135-156.

Walker, L. J. S., \& Healy, M. (1980). Psychological treatment of a burned child. Journal of Pediatric Psychology, 5(4), 395-404.

Weinberg, K., Birdsall, C., Vail, D., Marano, M. A., Petrone, S. J., \& Mansour, E. H. (2000). Pain and anxiety with burn dressing changes: Patient self-report. Journal Burn Care Rehabilitation, 21(2), 155-161.

Worden, J. W. (1987). Cognitive therapy with cancer patients. In A. Freeman, \& V. Greenwood (Eds.), Cognitive therapy: Applications in psychiatric and medical settings (pp. 80-87). New York: Human Sciences Press.

Yin, R. K. (2004). Estudo de caso: planejamento e métodos (3a ed). Porto Alegre: Artmed. 